\title{
Latitudinal Dependence of Wind-Induced Near-Inertial Energy
}

\author{
XIAOMING ZHAI \\ Centre for Ocean and Atmospheric Sciences, School of Environmental Sciences, University of East Anglia, \\ Norwich, United Kingdom
}

(Manuscript received 28 August 2015, in final form 7 October 2015)

\begin{abstract}
Midlatitude storms, accounting for the majority of wind energy input to near-inertial motions in the ocean, are known to shift their track significantly from one year to another. The consequence of such storm track shifts on wind-induced near-inertial energy (NIE) is yet unknown. Here, the latitudinal dependence of wind-induced NIE is first analyzed in the framework of the slab model and then tested using two numerical ocean models. It is found that the NIE input by pure inertial wind stress forcing, which dominates the wind energy input to near-inertial motions, is independent of latitude. As a consequence, the NIE generated by white-noise wind stress forcing is also latitudinally independent. In contrast, the NIE generated by red-noise wind stress forcing shows strong dependence on latitude owing to longer inertial periods at lower latitudes capable of sampling greater inertial wind stress forcing. Given that the observed surface wind stress spectra are red, results from this study suggest that an equatorward shift of the storm track is likely to result in an increase in wind-induced NIE in the ocean, while the opposite is true for a poleward shift.
\end{abstract}

\section{Introduction}

Near-inertial motions are believed to be an important energy source for generating diapycnal mixing in the ocean, contributing to the cooling of the sea surface temperature (e.g., Jochum et al. 2013) and maintenance of the global meridional overturning circulation against stable stratification (e.g., Wunsch and Ferrari 2004). The bulk of wind energy input to near-inertial motions in the ocean each year is typically accounted for by a handful of winter storms at midlatitudes (e.g., D'Asaro 1985). It is well known (e.g., Lau 1988) that the storm activity varies significantly from one year to another, not only in its intensity but also in its path. For example, in the North Atlantic, winter storm activity is closely linked to the North Atlantic Oscillation (NAO; e.g., Hurrell 1995; Rogers 1997). During positive NAO years, the storms tend to be more frequent and more intense, and the storm track stretches from Cape Hatteras northeastward toward the Nordic Seas, whereas during negative NAO years the storms tend to be less frequent and of reduced intensity, and the storm track

Corresponding author address: Xiaoming Zhai, School of Environmental Sciences, University of East Anglia, Norwich NR4 7TJ, United Kingdom.

E-mail: xiaoming.zhai@uea.ac.uk shifts equatorward and becomes more zonally oriented. Dippe et al. (2015) recently investigated the relationship between interannual variability of wind power input to near-inertial motions in the North Atlantic and the NAO and found greater basinwide wind power input during negative NAO years despite reduced storm intensity. They attributed this counterintuitive result to greater efficiency of storms in exciting near-inertial motions at lower latitudes. Storm tracks are also predicted to undergo changes in the future. For example, climate models project a consistent future intensification and poleward shift of the storm track in the Southern Hemisphere under global warming (e.g., Chang et al. 2012). The implication of these future storm track changes for wind-induced near-inertial energy (NIE) and its associated mixing is yet to be explored.

In this study, we investigate the latitudinal dependence of wind-induced NIE in the ocean, motivated by the observed and predicted storm track shifts. We first present some solutions based on a simple slab model to highlight the potential latitudinal dependence of wind-induced NIE and then test this latitudinal dependence using numerical ocean models driven by idealized wind forcing applied at various latitudes. The implications of storm track shifts on NIE are also discussed. 
(a)

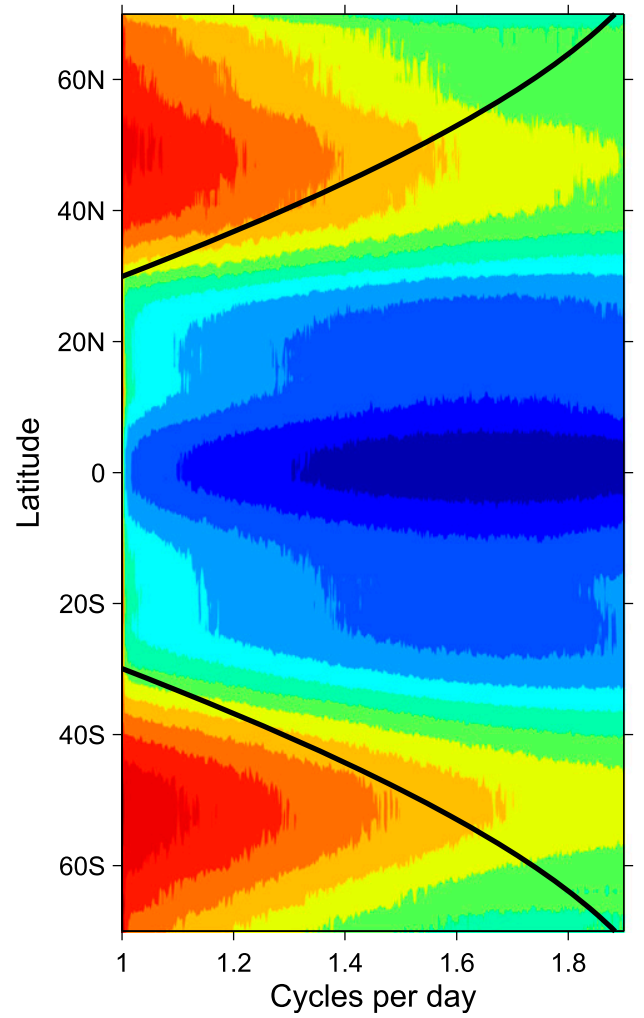

(b)

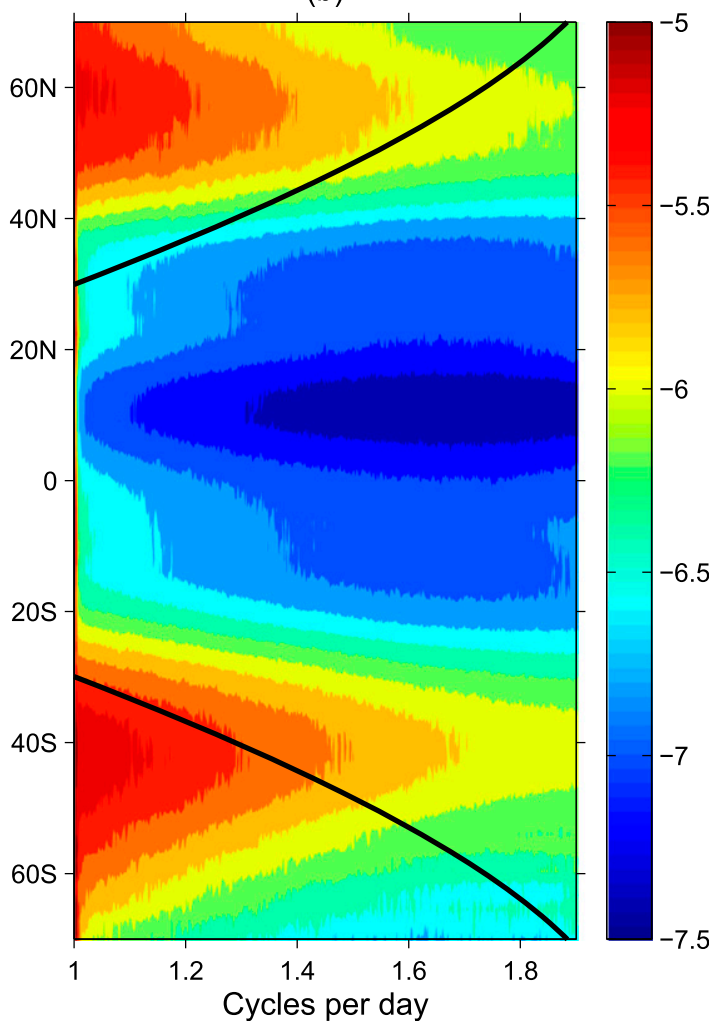

FIG. 1. (a) Zonally averaged surface wind stress spectra $\left(\mathrm{N}^{2} \mathrm{~m}^{-4}\right.$ per cycles per day) calculated from 6-hourly NCEP reanalysis wind stresses and averaged over the period of 2001-14. The black line corresponds to the local inertial period. (b) As in (a), but the whole wind stress field is shifted northward by $10^{\circ}$. Both (a) and (b) are plotted on a $\log$ scale with base 10 .

\section{Latitudinal dependence of NIE}

In this section the damped slab model of the mixed layer originally proposed by Pollard and Millard (1970) is used to highlight the latitudinal dependence of windinduced NIE in the ocean. The horizontal momentum equation of the slab model in complex form is given by

$$
\frac{\partial Z}{\partial t}+(\text { if }+r) Z=\frac{\mathcal{T}}{H},
$$

where $Z=u+i v$ is the horizontal current velocity, $\mathcal{T}=\left(\tau_{x}+i \tau_{y}\right) / \rho_{0}$ is the surface wind stress weighted by the reference density $\rho_{0}, f$ is the Coriolis parameter, $H$ is the thickness of the surface mixed layer, and $r^{-1}$ is the linear damping time scale that is usually set to be 2-10 days.

Assuming that $\mathcal{T}(t)=\mathcal{T}_{0} e^{-i \omega t}$, where $\omega$ is the frequency, (1) can be solved analytically, and its solution with the ocean in a state of rest as the initial condition is given by

$$
Z(t)=\frac{\mathcal{T}_{0}}{[i(f-\omega)+r] H}\left[e^{-i \omega t}-e^{-(i f+r) t}\right] .
$$

We now consider the following two limiting cases of wind forcing that are often discussed in the context of wind generation of near-inertial motions.

\section{a. Constant wind stress suddenly switched on}

Consider the situation where the ocean is initially at rest and then a constant zonal wind stress with amplitude $\mathcal{T}=\mathcal{T}_{0}$ is suddenly switched on (e.g., Gill 1982; Dippe et al. 2015). In this case, $\omega=0$ and the inertial component of (2) reduces to

$$
Z_{I}(t)=\frac{i \mathcal{T}_{0}}{f H} e^{-(i f+r) t},
$$

in the limit of $r \ll|f|$. The magnitude of the inertial currents in this case is inversely proportional to $|f|$, which means the same wind stress forcing that takes place at lower latitudes can excite stronger inertial currents. The NIE per unit area is given by

$$
\operatorname{KE}_{I}(t)=\rho_{0} H \frac{Z_{I}(t) Z_{I}^{*}(t)}{2}=\frac{\rho_{0} \mathcal{T}_{0}^{2}}{2 f^{2} H} e^{-2 r t} .
$$


(a)

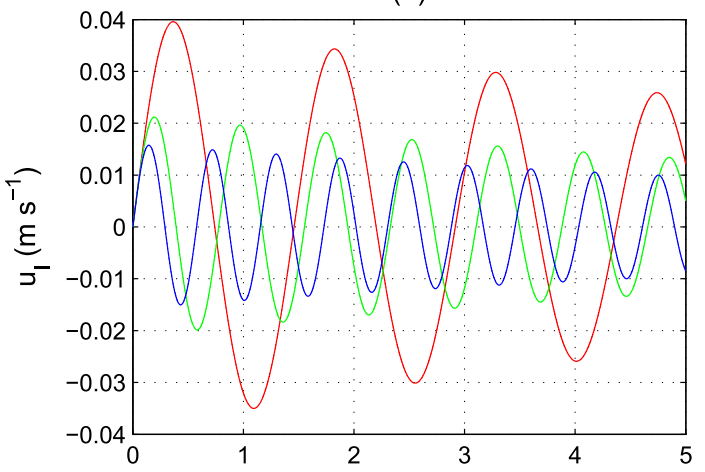

(c)

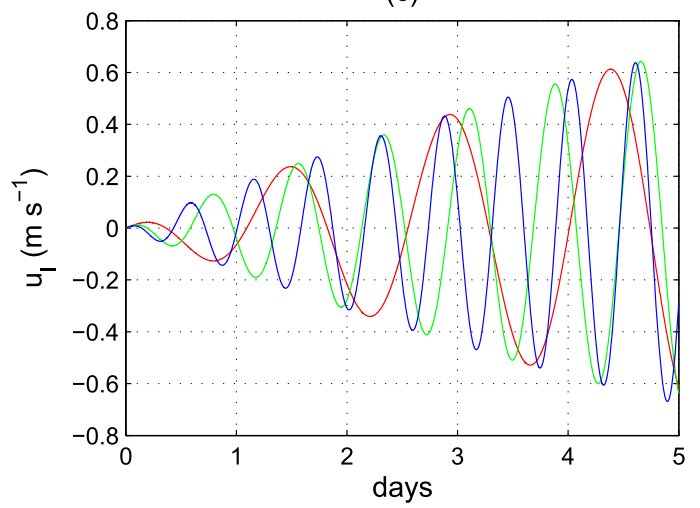

(b)

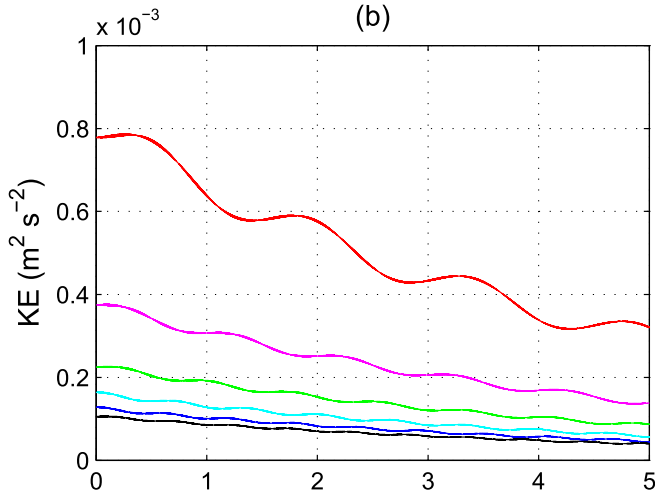

(d)

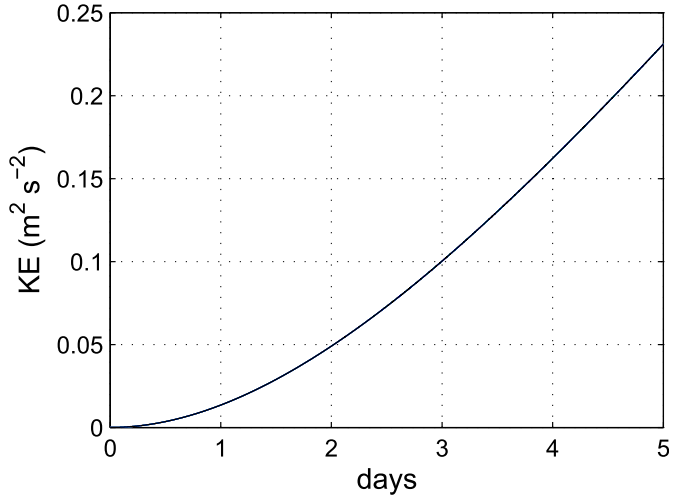

FIG. 2. Time series of the simulated (a) near-inertial zonal velocity and (b) NIE when the slab model is subject to a constant zonal wind stress being suddenly switched on at $20^{\circ} \mathrm{S}$ (red), $30^{\circ} \mathrm{S}$ (magenta), $40^{\circ} \mathrm{S}$ (green), $50^{\circ} \mathrm{S}\left(\mathrm{cyan}\right.$ ), $60^{\circ} \mathrm{S}$ (blue), and $70^{\circ} \mathrm{S}$ (black). (c),(d) As in (a),(b), but the slab model is forced by pure inertial wind stress. For clarity, only near-inertial zonal velocities at $20^{\circ}, 40^{\circ}$, and $60^{\circ} \mathrm{S}$ are plotted in (a) and (c). In (d) the six colored curves completely overlap each other such that only the black curve is visible. Note that the NIE in (d) is about two orders of magnitude greater than that in (b).

Equation (4) shows that the NIE decays at a rate of $2 r$ after being injected into the ocean at the instant the wind forcing is switched on. The net wind energy input to near-inertial motions $P_{I}$ is then

$$
P_{I}=\operatorname{KE}_{I}(t=0)=\frac{\rho_{0} \mathcal{T}_{0}^{2}}{2 f^{2} H}
$$

In this simple case of a constant wind stress being suddenly switched on, both the magnitudes of $\mathrm{KE}_{I}$ and $P_{I}$ are inversely proportional to $f^{2}$ and show strong latitudinal dependence. Equations (3)-(5) also suggest that the magnitude of the near-inertial response is inversely proportional to $H$. This sensitivity to $H$ results from the assumption in the slab model that $Z_{I}$ is mixed instantaneously over the prescribed mixed layer depth. However, a number of observational and modeling studies (e.g., Davis et al. 1981; Niiler and Paduan 1995; Crawford and Large 1996; Rath et al. 2014) have found that, at least for some wind events, $Z_{I}$ is not vertically homogeneous in the surface mixed layer, which renders $P_{I}$ less sensitive to $H$ than suggested by (5). Here we focus on the role of latitudinally varying $f$ in determining the magnitude of wind-induced NIE and assume $H$ is constant.

\section{b. Inertial wind stress forcing}

For the case of anticyclonically rotating inertial wind stress forcing where $\omega=f$, the inertial component of the solution to (1) is given by

$$
Z_{I}(t)=Z(t)=\frac{\mathcal{T}_{0}}{r H}\left(1-e^{-r t}\right) e^{-i f t},
$$

which, in the limit of small $r$, can be approximated by

$$
Z_{I}(t) \approx \frac{\mathcal{T}_{0} t}{H} e^{-i f t} .
$$

The NIE per unit area in this limit is then given by

$$
\mathrm{KE}_{I}(t)=\rho_{0} H \frac{Z_{I}(t) Z_{I}^{*}(t)}{2} \approx \frac{\rho_{0} \mathcal{T}_{0}^{2} t^{2}}{2 H},
$$




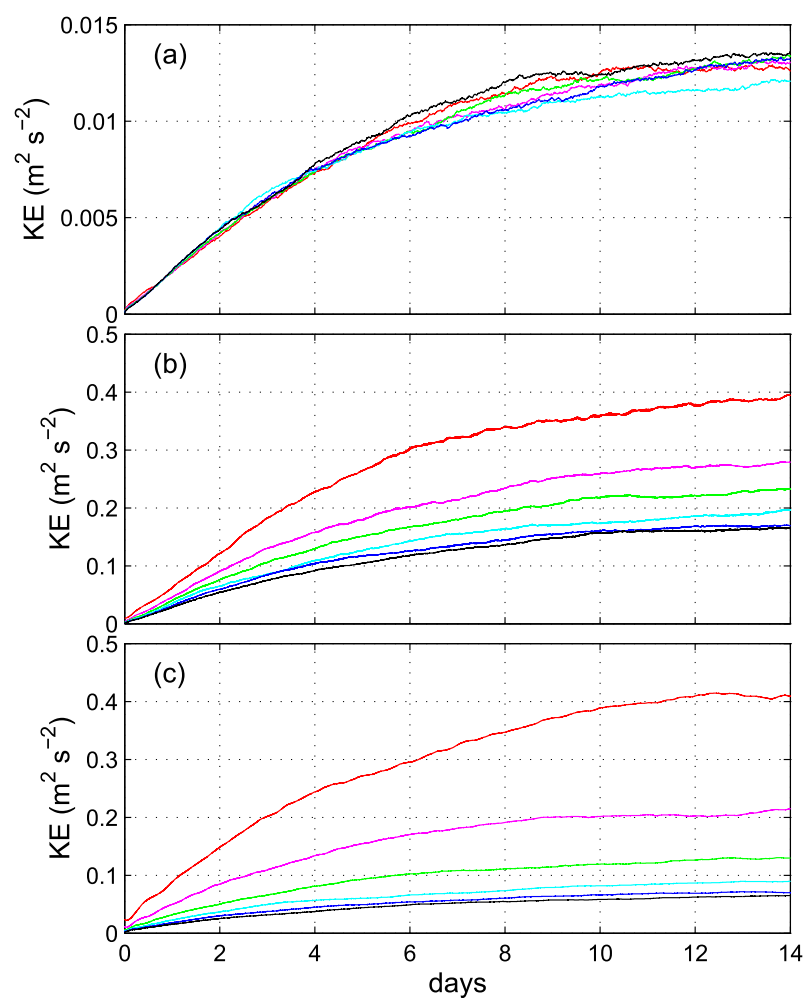

FIG. 3. Time series of the ensemble-averaged NIE at different latitudes in slab model experiments forced by the (a) white-noise wind stress, (b) red-noise wind stress with a spectral slope of -1 , and (c) red-noise wind stress with a spectral slope of -2 . The color labeling is the same as that in Fig. 2.

which is consistent with NIE input by the inertial wind forcing, that is,

$$
P_{I}(t)=\int_{0}^{t} \rho_{0} Z_{I}(t) \mathcal{T} *(t) d t \approx \frac{\rho_{0} \mathcal{T}_{0}^{2} t^{2}}{2 H} .
$$

This is the "inertial resonance" situation where both $\mathrm{KE}_{I}$ and $P_{I}$ grow rapidly with time because the wind stress vector in this case is constantly aligned with the near-inertial currents (e.g., Pollard and Millard 1970; D'Asaro 1985; Crawford and Large 1996). As a result, the inertial wind stress dominates the overall wind power input to near-inertial motions (Crawford and Large 1996). In the ocean, this growth in NIE will eventually be arrested by either shear instability and energy dissipation at the base of the mixed layer (e.g., Plueddemann and Farrar 2006; Zhai et al. 2009) or the intermittency of the inertial wind forcing events themselves (e.g., D'Asaro 1985). Importantly, (8) and (9) also show that, for the case of pure inertial wind stress forcing, both $\mathrm{KE}_{I}$ and $P_{I}$ are independent of latitude, in contrast to the situation where a constant wind stress is suddenly switched on.
However, this latitudinal independence of NIE works only if the magnitude of inertial wind stress forcing $\mathcal{T}_{0}$ is not a function of latitude, for example, white-noise wind stress forcing. The observed surface wind stress spectra, on the other hand, are red, with spectral slopes typically varying between -1 and -2 (e.g., Gille 2005). Figure 1a shows the global surface wind stress spectra averaged over the period 2001-14. Because the wind stress spectra are red and the inertial period decreases with latitude, longer inertial periods at lower latitudes are capable of sampling greater inertial wind stress forcing. In other words, an equatorward shift of the storm track will effectively result in an increase in the magnitude of the inertial wind stress forcing (Fig. 1b), that is, a greater $\mathcal{T}_{0}$, which then leads to greater $\mathrm{KE}_{I}$ and $P_{I}$. The opposite is true for a poleward shift of the storm track (Fig. 1b).

\section{Results from numerical experiments}

Solutions from the analytical slab model suggest that the wind-induced NIE may depend strongly on latitude. In this section, we test this latitudinal dependence using both a numerical slab model and a state-of-the-art primitive equation ocean model.

\section{a. Slab model}

The slab model is integrated numerically on an $f$ plane with $H$ and $r^{-1}$ set to be $50 \mathrm{~m}$ and 10 days, respectively. Four types of wind forcing are used here: 1) constant zonal wind stress of $0.1 \mathrm{~N} \mathrm{~m}^{-2}$ being suddenly switched on, 2) pure inertial wind stress with magnitude of $0.1 \mathrm{~N} \mathrm{~m}^{-2}, 3$ ) white-noise wind stress, and 4) red-noise wind stress. For each type of wind forcing, the slab model is run at six different latitudes, increasing poleward from $20^{\circ}$ to $70^{\circ} \mathrm{S}$ at an interval of $10^{\circ}$ latitude. To isolate the near-inertial response, a fifth-order Butterworth highpass filter with cutoff frequency of $0.8 f$ is applied to the horizontal velocities.

Figure 2 shows the time series of model-simulated near-inertial zonal velocity $\left(u_{I}\right)$ and NIE when the slab model is subject to the first two types of wind forcing at different latitudes. When the constant zonal wind stress is suddenly switched on, the NIE is injected initially into the slab model and afterward decays gradually with time. The amplitude of the near-inertial response in this case shows strong latitudinal dependence, with the NIE decreasing by a factor of 7 from $7.8 \mathrm{~cm}^{2} \mathrm{~s}^{-2}$ at $20^{\circ} \mathrm{S}$ to $1.1 \mathrm{~cm}^{2} \mathrm{~s}^{-2}$ at $70^{\circ} \mathrm{S}$ (Figs. 2a,b). When the model is forced by pure inertial wind stress, $u_{I}$ grows linearly and NIE grows quadratically with time at all latitudes, as predicted by analytical solutions. Importantly, neither $u_{I}$ nor NIE show any latitudinal dependence (Figs. 2c,d). 


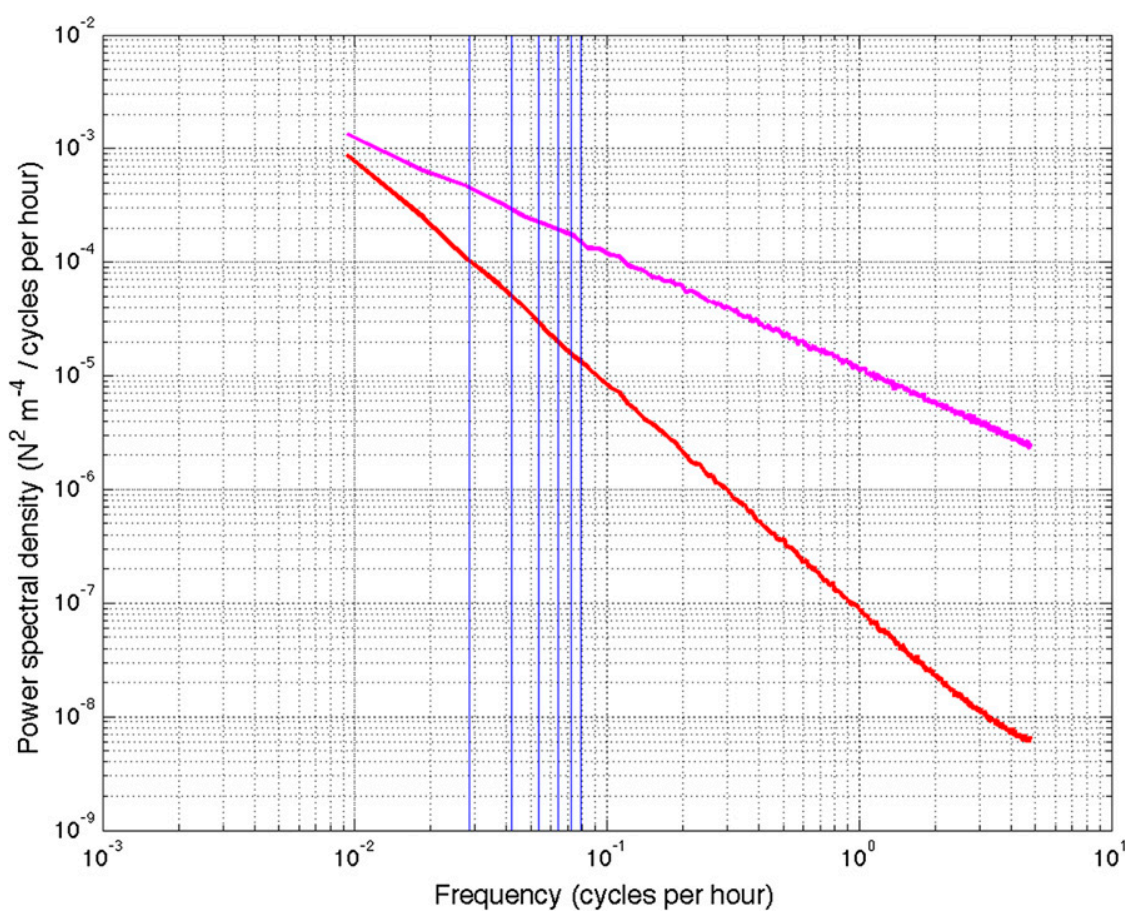

FIG. 4. Ensemble-averaged red-noise wind stress spectra. The pink and red curves have slopes of -1 and -2 , respectively. The vertical blue lines from left to right mark inertial frequencies at $20^{\circ}, 30^{\circ}, 40^{\circ}, 50^{\circ}, 60^{\circ}$, and $70^{\circ} \mathrm{S}$, respectively.

Note that the near-inertial response to pure inertial wind stress forcing is more than an order of magnitude greater than that to a constant wind stress that is suddenly switched on. This is apparently consistent with the notion that inertially rotating, "resonant" wind stress forcing is most efficient in transferring energy to near-inertial motions (e.g., Crawford and Large 1996).

For experiments with white-noise wind stress forcing, we conduct 1000 ensemble model simulations forced by 1000 time series of randomly generated white-noise zonal wind stress. The same procedure is also applied to the red-noise wind stress experiments. It is worth pointing out that, for a single ensemble model simulation, the NIE can peak at any latitude because of high intermittency of wind power input to near-inertial motions, and this is true even for the red-noise wind stress experiments (not shown).

Figure 3 shows the time series of ensemble-averaged NIE in experiments forced by the white-noise wind stress and red-noise wind stress with spectral slopes of -1 and -2 , respectively. In experiments forced by the white-noise wind stress, the ensemble-averaged NIE has essentially the same magnitude across all latitudes (Fig. 3a). This latitudinal independence of NIE in the white-noise wind stress experiments further confirms that the wind stress forcing at near-inertial frequencies dominates the overall wind energy input to near-inertial motions. In comparison, in experiments forced by the red-noise wind stress, there is a clear dependence of NIE on latitude; the amplitude of ensemble-averaged NIE decreases with latitude (Figs. 3b,c). The latitudinal dependence becomes even more pronounced when the slope of the wind stress spectra increases from -1 to -2 .

To understand where this latitudinal dependence comes about, in Fig. 4 we show the ensemble-averaged spectra of the red-noise wind stress forcing. It becomes immediately clear that the latitudinal dependence found in the red-noise wind stress experiments is due to longer inertial periods at lower latitudes capable of sampling greater inertial wind stress forcing. For example, the amplitude of the inertial wind stress forcing increases by a factor of $\sim 3$ from $70^{\circ}$ to $20^{\circ} \mathrm{S}$ when the slope of the wind stress spectra is -1 , but by a factor of $\sim 8$ when the slope further steepens to -2 .

\section{b. $M I T g \mathrm{~cm}$}

Although the slab model has been widely used for studying wind-induced NIE, it is worth emphasizing the limitations of such a simple model. For example, comparisons with observations show that the slab model may overestimate the wind energy input to near-inertial motions, particularly during strong wind events, owing to the lack of a dissipation mechanism acting on short time scales (e.g., Plueddemann and Farrar 2006). Here 
(a)

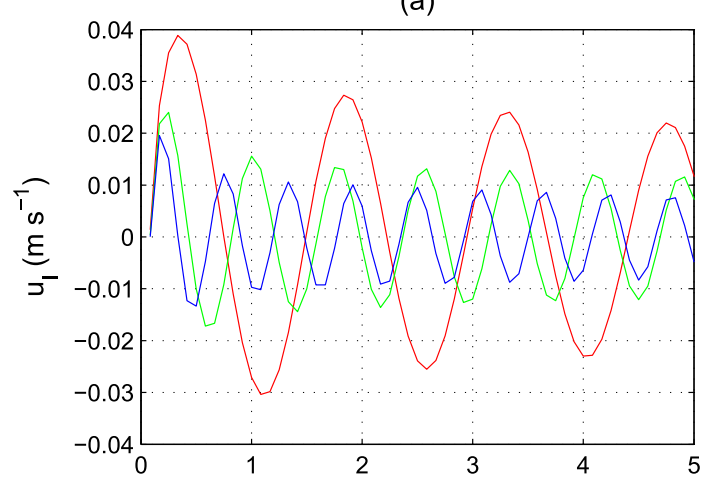

(c)

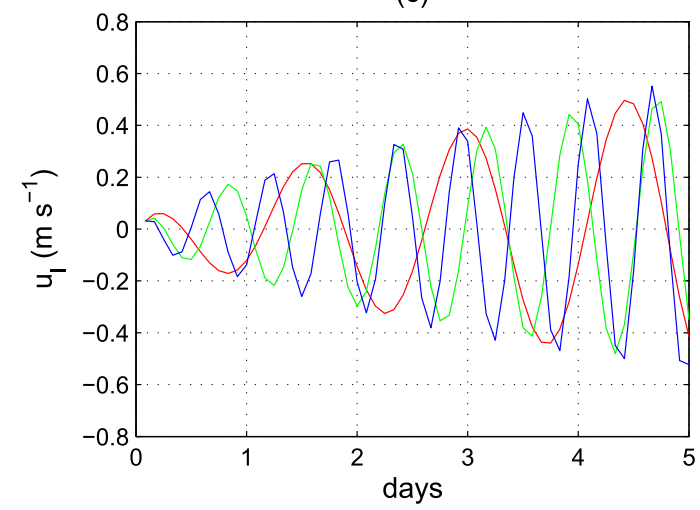

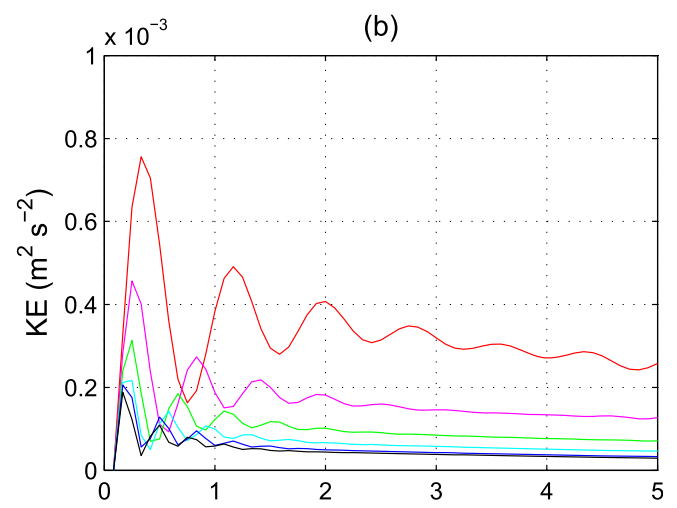

(d)

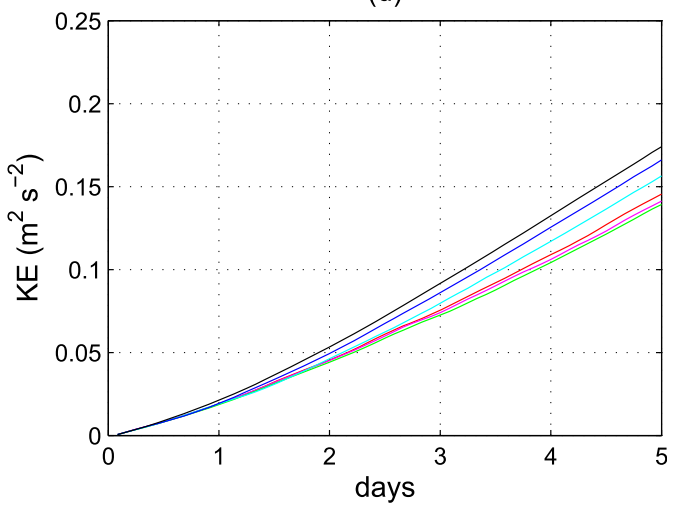

FIG. 5. As in Fig. 2, but for the MITgcm experiments.

we repeat the above slab model experiments using the MIT general circulation model (MITgcm; Marshall et al. 1997). The model domain is chosen to be a zonally reentrant channel on an $f$ plane that is $2400 \mathrm{~km}$ in zonal extent, $1200 \mathrm{~km}$ in meridional extent, and $2000 \mathrm{~m}$ deep with a flat bottom. The horizontal grid spacing is $6 \mathrm{~km}$. There are 50 geopotential levels whose thickness increases with depth, ranging from $5 \mathrm{~m}$ in the top $50 \mathrm{~m}$ to $150 \mathrm{~m}$ near the bottom. Sponges are applied at the southernmost and northernmost regions of the model domain to damp out any waves approaching these boundaries. We employ the K-profile vertical mixing parameterization (Large et al. 1994). The model is initialized with a vertical temperature profile that is uniform in the top $50 \mathrm{~m}$ and decays exponentially below that.

Figure 5 shows that the MITgcm reproduces the salient features of the slab model when subject to the first two types of wind stress forcing, that is, strong dependence of NIE on latitude when forced by the same constant wind stress being suddenly switched on (Figs. 5a,b) and independent of latitude when forced by pure inertial wind stress (Figs. 5c,d). There is some divergence among NIE at different latitudes in Fig. 5d, most likely because of the mixing scheme used in the
MITgcm, whereas in the slab model the damping coefficient $r$ is simply set to be a constant. For experiments with stochastic wind stress forcing, we conduct 30 ensemble MITgcm model runs forced by randomly generated white-noise wind stress and another 30 ensemble model runs forced by red-noise wind stress that has a spectral slope of -2 . Consistent with the results from the slab model, the ensemble-averaged NIE in experiments forced by the white-noise wind stress shows no clear dependence on latitude (Fig. 6a), with the ensembleand time-averaged NIE at $20^{\circ}, 40^{\circ}$, and $60^{\circ} \mathrm{S}$ all close to $0.018 \mathrm{~m}^{2} \mathrm{~s}^{-2}$. The ensemble-averaged NIE in experiments forced by the red-noise wind stress (Fig. 6b), on the other hand, shows strong latitudinal dependence, with the ensemble- and time-averaged NIE at $20^{\circ} \mathrm{S}$ almost double that at $40^{\circ} \mathrm{S}$ and triple that at $60^{\circ} \mathrm{S}$, again confirming the results from the slab model experiments.

\section{Concluding remarks}

The latitudinal dependence of wind-induced NIE has been investigated analytically in the framework of the slab model and tested numerically using both a numerical slab model and the MITgcm. Our main findings are as follows: 

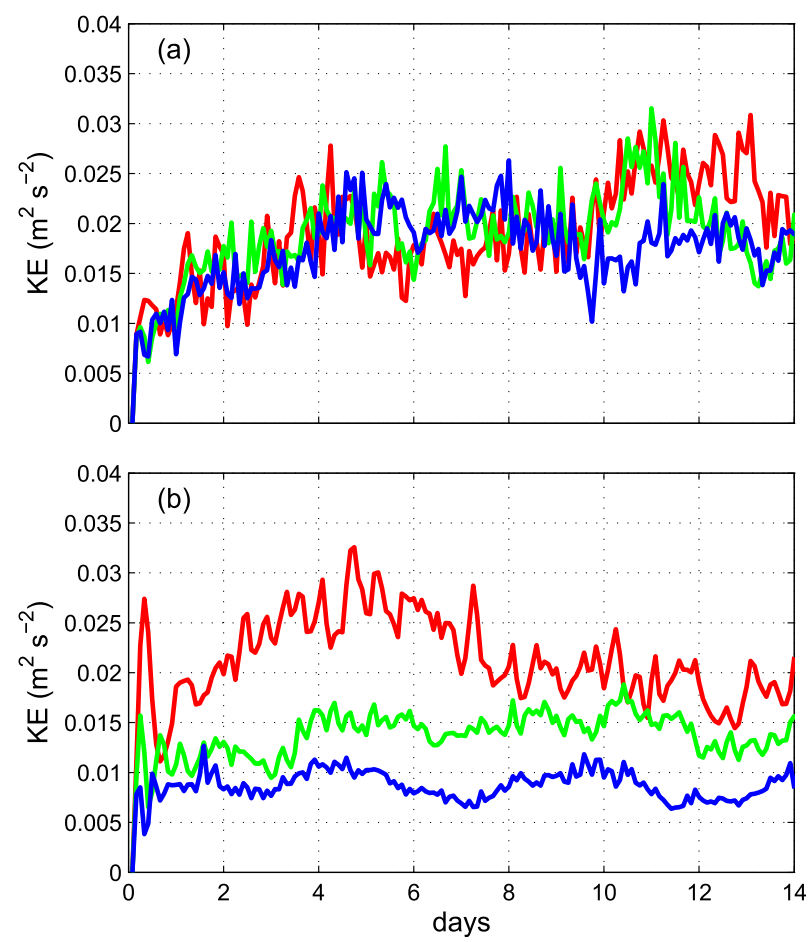

FIG. 6. Time series of the ensemble-averaged NIE at $20^{\circ} \mathrm{S}$ (red), $40^{\circ} \mathrm{S}$ (green), and $60^{\circ} \mathrm{S}$ (blue) in MITgcm experiments forced by the (a) white-noise wind stress and (b) red-noise wind stress with a spectral slope of -2 .

- The NIE excited by constant wind stress being suddenly switched on is inversely proportional to $f^{2}$ and therefore shows strong latitudinal dependence.

- The NIE input by pure inertial wind stress forcing, which dominates wind energy input to near-inertial motions, is independent of latitude. As a result, the NIE generated by white-noise wind stress forcing is also latitudinally independent.

- The NIE generated by red-noise wind stress forcing depends strongly on latitude because longer inertial periods at lower latitudes effectively sample greater inertial wind stress forcing.

Given that the observed surface wind stress spectra are red (Fig. 1; Gille 2005), results from our study suggest that an equatorward shift of the storm track is likely to result in an increase in wind-induced NIE in the ocean, while the opposite is true for a poleward shift. Variations of the surface mixed layer depth with latitude may also contribute to changes in NIE when the storm track shifts, but this issue is not explicitly treated here and left for a future study, as it requires an improved understanding of the vertical structure of the near-inertial currents in response to various wind forcing events (e.g., Rath et al. 2014). Finally, it is worth emphasizing that the latitudinal (in)dependence of NIE induced by stochastic wind stresses only emerges after ensemble averaging owing to high intermittency of strong inertial wind forcing events.

Acknowledgments. I wish to thank Tina Dippe, Richard Greatbatch, and Willi Rath for helpful discussions, and I thank two anonymous reviewers for their constructive comments that led to an improved manuscript.

\section{REFERENCES}

Chang, E. K. M., Y. Guo, and X. Xia, 2012: CMIP5 multimodel ensemble projection of storm track change under global warming. J. Geophys. Res., 117, D23118, doi:10.1029/ 2012JD018578.

Crawford, G. B., and W. G. Large, 1996: A numerical investigation of resonant inertial response of the ocean to wind forcing. J. Phys. Oceanogr., 26, 873-891, doi:10.1175/1520-0485(1996)026<0873: ANIORI $>2.0 . \mathrm{CO} ; 2$.

D'Asaro, E. A., 1985: The energy flux from the wind to nearinertial motions in the surface mixed layer. J. Phys. Oceanogr., 15, 1043-1059, doi:10.1175/1520-0485(1985)015<1043: TEFFTW $>2.0 . \mathrm{CO} ; 2$.

Davis, R. E., R. deSzoeke, D. Halpern, and P. Niiler, 1981: Variability in the upper ocean during MILE. Part I: The heat and momentum balances. Deep-Sea Res., 28, 1427-1451, doi:10.1016/0198-0149(81)90091-1.

Dippe, T., X. Zhai, R. J. Greatbatch, and W. Rath, 2015: Interannual variability of wind power input to near-inertial motions in the North Atlantic. Ocean Dyn., 65, 859-875, doi:10.1007/ s10236-015-0834-x.

Gill, A. E., 1982: Atmosphere-Ocean Dynamics. Academic Press, $662 \mathrm{pp}$.

Gille, S. T., 2005: Statistical characterization of zonal and meridional ocean wind stress. J. Atmos. Oceanic Technol., 22, 13531372, doi:10.1175/JTECH1789.1.

Hurrell, J. W., 1995: Decadal trends in the North Atlantic Oscillation and relationships to regional temperatures and precipitation. Science, 269, 676-679, doi:10.1126/ science.269.5224.676.

Jochum, M., B. P. Briegleb, G. Danabasoglu, W. G. Large, N. J. Norton, S. R. Jayne, and F. O. Bryan, 2013: The impact of oceanic near-inertial waves on climate. J. Climate, 26, 28332844, doi:10.1175/JCLI-D-12-00181.1.

Large, W. G., J. C. McWilliams, and S. C. Doney, 1994: Oceanic vertical mixing: A review and a model with a nonlocal boundary layer parmeterization. Rev. Geophys., 32, 363-403, doi:10.1029/94RG01872.

Lau, N. C., 1988: Variability of the observed midlatitude storm tracks in relation to low-frequency changes in the circulation pattern. J. Atmos. Sci., 45, 2718-2743, doi:10.1175/ 1520-0469(1988)045<2718:VOTOMS > 2.0.CO;2.

Marshall, J., A. Adcroft, C. Hill, L. Perelman, and C. Heisey, 1997: A finite-volume, incompressible Navier Stokes model for studies of the ocean on parallel computers. J. Geophys. Res., 102, 5753-5766, doi:10.1029/96JC02775.

Niiler, P., and J. D. Paduan, 1995: Wind-driven motions in the northeast Pacific as measured by Lagrangian drifters. J. Phys. Oceanogr., 25, 2819-2830, doi:10.1175/1520-0485(1995)025<2819: WDMITN $>2.0 . \mathrm{CO} ;$. 
Plueddemann, A. J., and J. T. Farrar, 2006: Observations and models of the energy flux from the wind to mixed-layer inertial currents. Deep-Sea Res. II, 53, 5-30, doi:10.1016/ j.dsr2.2005.10.017.

Pollard, R. T., and R. C. J. Millard Jr., 1970: Comparison between observed and simulated wind-generated inertial oscillations. Deep-Sea Res. Oceanogr. Abstr., 17, 813-821, doi:10.1016/ 0011-7471(70)90043-4.

Rath, W., R. J. Greatbatch, and X. Zhai, 2014: On the spatial and temporal distribution of near-inertial energy in the Southern Ocean. J. Geophys. Res. Oceans, 119, 359-376, doi:10.1002/ 2013JC009246.
Rogers, J. C., 1997: North Atlantic storm track variability and its association to the North Atlantic Oscillation and climate variability of northern Europe. J. Climate, 10, 1635-1647, doi:10.1175/1520-0442(1997)010<1635: NASTVA $>2.0 . \mathrm{CO} ; 2$.

Wunsch, C., and R. Ferrari, 2004: Vertical mixing, energy, and the general circulation of the oceans. Annu. Rev. Fluid Mech., 36, 281-314, doi:10.1146/annurev.fluid.36.050802.122121.

Zhai, X., R. J. Greatbatch, C. Eden, and T. Hibiya, 2009: On the loss of wind-induced near-inertial energy to turbulent mixing in the upper ocean. J. Phys. Oceanogr., 39, 3040-3045, doi:10.1175/2009JPO4259.1. 